\title{
PERSONAJES FICTICIOS Y NOMBRES VACÍOS: UNA PERSPECTIVA ONTOLÓGICA DESINFLACIONARIA DESDE LA REFERENCIA DIRECTA
}

\section{Nicolás Alarcón Z*}

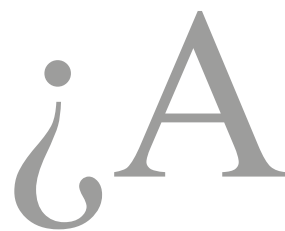

Qué nos Referimos CuAndo sostenemos Que Pegaso tiene dos ALAs? Parece obvio que el enunciado tiene sentido, pero fallamos al señalar el referente. Una forma de entender este tipo de enunciados, los cuales poseen personajes ficticios o nombres vacíos como sujetos, es recurrir a la teoría de Meinong. En lo presente, presentaré una serie de objeciones a dicha perspectiva inflacionaria, en pos de una resolución ontológicamente mínima que respete nuestras intuiciones.

Palabras claves: Meinong, personajes ficticios, nombres vacíos, teorías de la referencia, referencia directa.

\section{FICTIONAL CHARACTERS AND EMPTY NAMES: A DEFLATONARY ONTOLOGICAL PERSPETIVE FROM DIRECT REFERENCE}

What do we mean when we say "Pegasus has two wings"? It seems obvious that this statement makes sense, although it has not an existent referent. One way of understanding statements containing fictional characters or empty names as subjects is through Meinong's theory. In the present article, I will present some objections to Meinong's ontologically inflationary perspective. The goal of these arguments is to offer an ontologically minimal resolution, consistent with our intuitions, to the problem of non-existent referents.

Keywords: Meinong, fictional characters, vacuum names, reference theory, direct reference.

\footnotetext{
* Licenciado en Filosofía; Dr.(c) en Filosofía, Universidad Alberto Hurtado.nico.az626@gmail.com
} 


\section{El problema}

(1) Hamlet fue un melancólico príncipe danés. A pesar de que entendemos el sentido de dicho enunciado -entendemos que se está predicando la melancolía de un sujeto, esto es, de Hamlet- cuando salimos a buscar el referente del predicado, simplemente no lo encontramos. Aun así, si un profesor pregunta a sus alumnos si es verdad que Hamlet fue un melancólico príncipe danés, es muy probable que aquel que responda que tal enunciado es falso, obtenga una mala calificación.

Lo anterior abre un dilema: ¿cómo podemos sostener que tal enunciado es verdadero, si el sujeto no posee un referente? De forma más específica, el problema se puede plantear de la siguiente forma: ¡cómo es posible que podamos hacer enunciados verdaderos, cuyos sujetos sean entidades ficticias, personajes ficticios, nombres vacíos, en suma, que no posean referente? En resumen, cuando nos preguntamos sobre el referente de dichos enunciados -sobre Hamlet y su melancolía- nos estamos preguntando sobre el estado ontológico de entidades como Frodo Bolsón, Eddard Stark, El Viejo Pascuero, etc; en resumen, preguntamos sobre entidades ficticias [EF] (o como otro los llaman: nombres vacíos').

En un marco cotidiano, tenemos la intuición de que, enunciados con EF como sujetos, son verdaderos. En cuanto a las EF, creemos, ingenuamente, que "existen" de alguna manera; claro está, que dicha "existencia" la tomamos en un sentido débil, sosteniendo que tales entidades no están en el espacio, como sí estamos nosotros. ¿Cómo lidiamos con tal intuición? ¿Cómo conciliar el que creamos que los enunciados son verdaderos, frente al hecho obvio que los EF no poseen referente?

1 Asumiré y usaré, a lo largo de la presente investigación, que entidades ficticias y nombres vacíos se refieren a lo mismo, esto es: nombres propios tales como Hamlet, que hacen referencia a entidades que i) son parte de una obra literaria y ii) que no podemos encontrar en la realidad. Añado está última clausula, pues puede ser el caso que encontremos una obra literaria cuyo protagonista sea una entidad real, Napoleón, por ejemplo -en tal situación el problema no se presenta-o al menos no de la misma manera, pues Napoleón sí posee un referente.

Asimismo, y a modo de aclaración y delimitación, no me referiré al problema del estatus ontológico de entidades matemáticas. Si bien las usaré de ejemplo en algunos casos, no me referiré al problema concerniente a su existencia. Si bien se puede tomar como una situación análoga la de Hamlet con la del número 2, no extenderá las posibles conclusiones y consecuencias de la presente investigación a tal campo. Tomaré, por tanto, la discusión sobre la realidad de las entidades matemáticas como independientes de esta. 
Frente a tales interrogantes respecto al estado ontológico de EF han surgido distintas opciones, una de ellas, dada por Alexius Meinong en su Theory of Objects $^{2}$; de esta se desprende que las entidades implicadas en tales enunciados poseen existencia, pero en un sentido amplio del término existencia. Tal teoría respeta nuestras intuiciones, pero pagando un costo elevado.

En lo que sigue, buscaré plantear la solución ofrecida por Meinong respecto a EF, señalando sus virtudes y debilidades, además de recurrir a la crítica ofrecida por Russell respecto a la idea de subsistencia. Finalmente, expondré la solución dada por Kripke y su teoría de la referencia directa al problema de EF. Lo que me interesa defender de fondo, es resolver cierta tensión que se genera en dos ámbitos: semántico y ontológico. Para ser más preciso, busco dar con una propuesta que respete nuestra intuición semántica sobre enunciados similares a (1), a saber, que son enunciados verdaderos, pero que no nos comprometa con una ontología inflacionaria (esto es, con la existencia independiente de EF).

\section{Meinong y La Subsistencia}

Meinong sostenía que hay objetos de los cuales es verdad que no hay tales objetos ${ }^{3}$. Desde los números y todas las entidades matemáticas, hasta objetos tan peculiares y contradictorios como los círculos cuadrados, tienen cabida en la ontología de Meinong. Si bien, se le puede criticar -y con razón- lo sobrepoblado que concebía el universo, no se le puede alegar su principal intuición: tales objetos no son una mera nada. Nuestro prejuicio hacia lo real hace que dejemos fuera una infinidad de objetos posibles, los cuales usamos en nuestra vida cotidiana. Es en este sentido que se sostiene que Meinong es un realista de EF, pues les adjudica existencia.

En términos generales, el argumento de Meinong a favor de tales objetos -y en contra de lo que denominaba el "prejuicio de lo real"- se puede esquematizar de la siguiente manera ${ }^{4}$ :

2 MEINONG, Alexius. "The Theory of Objects". Traducción de Isaac Levi, D. B. Terrel, y Roderick Chisholm; en Realism and the Background of Phenomenology, ed. Roderick Chisholm. Ridgeview, 1981, pp. 76-117.

3 Cfr: MEINONG, Alexius. "The Theory of Objects". Traducción de Isaac Levi, D. B. Terrel, y Roderick Chisholm; en Realism and the Background of Phenomenology, ed. Roderick Chisholm. Ridgeview, 1981, p 83.

4 Cfr: CHISHOLM, Roderick. "Beyond Being and Nonbeing"; en Philosophical Studies: An International Journal for Philosophy in the Analytic Tradition, Vol. 24, No. 4, 1973, pp. 246-247 
p1: ciertos objetos, que no existen, tienen ciertas propiedades.

p2: hay objetos reales que no existen.

p3: no es el caso que un objeto tendrá propiedades, si, y solo si, este es real. c: la totalidad de los objetos se extiende más allá de lo real.

Tomemos, por ejemplo, el enunciado (1). De él se desprende que hay un "objeto" -Hamlet- el cual posee ciertas cualidades - ser melancólico, ser príncipe, ser danés- pero, además, sabemos que Hamlet no existe. ¿Cómo es posible, además de ser un enunciado verdadero, que Hamlet pueda instanciar propiedades? La respuesta la podemos obtener del argumento anterior: las propiedades no se limitan a objetos existentes, sino que se aplican a todos los objetos reales, los cuales incluyen objetos que no existen (como Hamlet).

Esto es posible para Meinong, pues interpreta la no-existencia del objeto intencional de Brentano de una forma particular'; cree que tales objetos no se encuentran necesariamente en el espacio-tiempo, pero no por ello carecen de una existencia en un sentido amplio. A continuación, caracterizaré dos nociones importantes de la ontología meinongiana, para luego analizar el caso particular de las entidades ficticias.

\section{A. Dos nociones de existencia}

La tesis fundamental de Meinong, siguiendo a Chisholm ${ }^{6}$, se puede sintetizar en dos grandes componentes: i) hay objetos que no existen, y ii) esos objetos que no existen están constituidos de manera tal que pueden ser sujetos de predicaciones verdaderas. Lo que se anuncia en ii) es la denominada doctrina del Aussersein, o de indiferencia al ser; el objeto es indiferente al ser y la existencia, pues al momento de caracterizarlo basta con poder dar ciertos rasgos del mismo, sin necesidad de sostener si este existe o no. De esta manera, se puede hacer declaraciones verdaderas acerca de objetos que no existen: el número 2 es un número par, sin embargo, tal objeto no existe

5 MEINONG, Alexius. "Über die Erfahrungsgrundlagen unseres Wissens", en Abhandlungen zur Didaktik und Philosophie der Naturwissenschaften, Band [Vol.] I, Heft [Issue] 6, Berlin: J. Springer. Reimpreso en Meinong 1968-78, Vol. V: 367-481. Epistemological treatise on empirical knowledge - on internal and external perception in contrast to a priori knowledge, \$10, 54-57. Revisado por Russell, 1906.

6 CHISHOLM, Roderick. (1973). "Beyond Being and Nonbeing”; en Philosophical Studies: An International Journal for Philosophy in the Analytic Tradition, Vol. 24, No. 4, 1973, pp. 246-257. 
But, though every object may correctly be said to be something or other, it is not the case that every object may correctly be said to be. Unicorns, golden mountains, and round squares may not be said to be at all. Everything, however, is an object, whether or not it exists or has any other kind of being, and indeed whether or not it is even thinkable. (Whatever is unthinkable, after all, at least has the property of being unthinkable.) And every object, clearly, has the characteristics it does have whether or not it has any kind of being. This last is the proposition Mally expressed by saying that the Sosein of an object is independent of its $\mathrm{Sein}^{7}$

Lo que importa, cuando señalamos un objeto, es poder caracterizarlo de cierta forma; esto es, cada objeto tiene un Sosein, o un conjunto de características entre las cuales no se encuentra la existencia. De esta forma, cuando hablamos de un unicornio, no podemos decir que existen propiamente tal, pero al menos sí podemos sostener que posee tal y tal característica. Lo mismo ocurre con Hamlet en el enunciado (1): este no existe, pero sí posee un Sosein, y por tanto puede ser caracterizado. Sin embargo, hay un sentido en el cual se sostiene que unicornios, números, Hamlet y otras entidades, no existen. En este punto se debe resaltar que hay, a lo menos, dos sentidos de existencia para Meinong: existencia en un sentido amplio [EA] y existencia en un sentido estrecho [EE]

- EE: un ítem existirá, si y solo si, este ocupa un lugar en el espacio-tiempo ${ }^{8}$.

- EA: un ítem existirá, si y solo si, se le puede adscribir ciertas cualidades, rasgos, propiedades, etc.

Es en este último sentido, EA, donde se habla de subsistencia. Un objeto x podrá no estar ocupando un lugar en un espacio-tiempo, pero al menos tiene un Sosien que permite que sea caracterizado, pudiendo subsistir, pero no existir en un sentido pleno.

\section{b. Personajes ficticios: ¿entre el Ser y el no Ser?}

Cuando realizamos enunciados como (1), o como (2) los unicornios tienen un cuerno, lo que hacemos es dar ciertas características de un objeto. Tales objetos, en tanto son susceptibles de ser descritos, caen dentro del criterio EA. En otras palabras,

7 Ibídem, p. 246. Mí énfasis

8 Ídem 
Hamlet, unicornios y toda entidad EF no existen en un sentido estrecho, pero no por ello son una mera nada; poseen un Sosein, poseen determinaciones y están sujetos a predicaciones verdaderas.

Entonces, el enunciado (1) sería verdadero, aunque el sujeto implicado -Hamlet- no existe, sino que subsiste. Esto salvaría nuestra intuición más básica, a saber, que i) tales enunciados tienen un sentido y ii) pueden ser verdaderos.

Si lo anterior es cierto, si Meinong está en lo correcto, no tendríamos mayores problemas con EF, y la presente investigación habría llegado a su fin; después de todo, se habrían respondido las preguntas que nos interesan: hace justicia a nuestra intuición, sosteniendo que enunciados como (1) son verdaderos. Sin embargo, para ello, deberíamos obviar los problemas que implica la propuesta de Meinong.

Un problema obvio, es que la teoría completa genera una jungla ontológica. Como ya lo señalaba Chisholm: "everything, however, is an object, whether or not it exists or has any other kind of being, and indeed whether or not it is even thinkable". Entre aquellos objetos encontramos, incluso, entidades contradictorias como el círculo cuadrado. Esto solo mantiene el problema original, expandiendo nuestra ontología al incluir EF como existentes.

\section{El caso de Russell contra Meinong}

Si el parámetro usado por Meinong para que un objeto entre en su ontología es que pueda ser descrito, no sería extraño encontrar objetos como círculos cuadrados. Que su Sosein sea contradictorio no es un impedimento para que califique como objeto.

Esta violación al principio del tercer excluido, es lo que lleva a Bertrand Russell ${ }^{10} \mathrm{a}$ criticar la propuesta de Meinong. Gruesamente, la propuesta que da en On Denoting (1905), se basa en al menos tres ideas: i) la existencia no es un predicado, sino una función de segundo orden, ii) los nombres propios son descripciones escondidas asociadas a un sujeto, y iii) los nombres lógicos serían los correspondientes a un sense-data -esto, eso- .

9 Ídem

10 RUSSELL, Bertrand. “On Denoting”; en Mind, Mind, New Series, Vol. 14, No. 56., 1905, pp. 479-493 
Although it is a fact that the existent present King of France/the round square does not exist, one also has to conclude (...) that he/it does exist. Russell thinks he can provide a radical cure for Meinong's inconsistencies by applying his theory of descriptions, which treat definite (and indefinite) descriptions as incomplete symbols that are to be eliminated in favor of existential quantification and predicates. According to Russell, the problems involved stem from the mistaken view that the grammatical form of language always corresponds to its logical form, and that if an expression means something, there must always be something that it means. But while denoting phrases such as "The so-and-so" look like referring expressions they are, in fact, nothing of the sort. It is because Meinong wrongly assumes that such expressions are referring expressions whose meaning is what they refer that his theory is apt to infringe logical laws ${ }^{11}$

Los nombres propios como Aristóteles son, en realidad, descripciones definidas [DD] disfrazadas. La idea de DD es poder señalar un individuo, y solo un individuo, que cumple con la descripción ${ }^{12}$. De esta forma, la existencia sería poder satisfacer tal función ${ }^{13}$. Entonces, el nombre Aristóteles sería en realidad una DD, como "el maestro de Alejandro Magno" y cuando sostenemos que Aristóteles existió, sostenemos que hay un individuo que cumple con tal característica. Formalmente, DD se expresa de la siguiente manera:

$$
\text { [DD] } \exists x((F x \& G x) \&(\forall y)(F y \rightarrow x=y))
$$

Entonces, si tomamos enunciados del tipo (3) Aristóteles amaba a los perros, en realidad estamos diciendo que (4) el maestro de Alejandro Magno amaba a los perros; la formalización representa la idea de que

i) hay a lo menos un individuo que es $\mathrm{F}$

ii) hay a lo sumo un individuo que es $\mathrm{F}$

iii) ese individuo que es F, también es $\mathrm{G}$.

11 MAREK, Johann. Alexius Meinong., 2013, disponible en http://plato.stanford.edu/archives/fall2013/entries/meinong/

12 RUSSELL, Bertrand. “On Denoting”; en Mind, Mind, New Series, Vol. 14, No. 56., 1905, p 488

13 Cfr: KRIPKE, Saul. "Vacuous Names and Fictional Entities", Oxford university Press, 2011, p. 53; y de nuevo en KRIPKE, Saul. "Reference and Existence: The John Locke Lectures". Oxford University Press, 2013, pp 6-7 
Este principio busca que, ciertos enunciados que violan el principio del tercer excluido, posean un valor de verdad definido. Adicionalmente, permite una ontología desinflacionaria, pues DD busca escoger un individuo que cumpla con los predicados estipulados; si no hay una entidad que cumpla con la exigencia, entonces no existe. Entonces, si tomamos enunciados que contengan EF como (5) Hamlet desconfía de su tío, y reemplazamos el nombre Hamlet por alguna DD asociada tal como "melancólico príncipe danés", obtendremos (6) el melancólico príncipe danés desconfía de su tío. En términos de DD, (6) sería claramente falsa, pues no describe nada que de hecho exista. En otras palabras, no hay entidad que cumpla las condiciones de verdad exigidas por DD en (6), a diferencia de (4) donde si encontramos un individuo que cumple las condiciones exigidas.

Según Meinong, enunciados como (5) serían semejantes -en términos de valor de verdad- a enunciados del tipo (3): ambos serían enunciados verdaderos. Russell no sólo cree que (5) es falsa, sino que se debería analizar de manera distinta. Si bien, la propuesta de Russell es tentadora ontológicamente -nos otorga una ontología desinflacionaria- pasa a llevar nuestras intuiciones respecto a enunciados que involucran EF.

Siempre puede ser el caso que nuestras intuiciones estén equivocadas y, si ese es el caso, Russell tiene toda la razón al sostener que (5) -y cada enunciado que contenga un EF- es falsa. Sin embargo, y como nota Chisholm ${ }^{14}$, sí hay un caso a favor de Meinong que Russell parece no notar, pues sí parecen haber muchas verdades respecto a objetos tales que no hay tales objetos ${ }^{15}$. En el caso de EF, estos parecen ser objetos intencionales ${ }^{16}$, de los cuales no tiene mucho sentido sostener si los enunciados son falsos. Si tengo una actitud intencional hacia un objeto inexistente $\mathrm{X}$, no tiene sentido sostener que tal actitud es falsa

Russell's procedure would provide us with a statement that is false ("There exists an $\mathrm{x}$ such that $\mathrm{x}$ is a mountain $\mathrm{I}$ am thinking of and $\mathrm{x}$ is golden, and, for every $\mathrm{y}$, if $\mathrm{y}$ is a mountain I am thinking of, then $\mathrm{y}$ is identical with $\mathrm{x}$ ") ${ }^{17}$

14 CHISHOLM, Roderick. (1973). "Beyond Being and Nonbeing", Philosophical Studies: An International Journal for Philosophy in the Analytic Tradition, Vol. 24, No. 4, 1973, pp. 245-257

Cfr: Ibidem, p 248

16

Cfr: Ibídem,p 251

17 Ibídem, p. 253. 
Si tenemos enunciados sobre EF, de los cuales DD no puede dar cuenta, parece que la propuesta de Russell no es del todo satisfactoria.

Si bien DD responde a uno de los requerimientos - no conceder existencia a EF- no es capaz de hacer justicia a nuestra intuición sobre tales enunciados. Siempre puede ser el caso que estemos equivocados, pero nuestras prácticas lingüísticas parecen indicar lo contrario. Si alguien me pregunta "¿es cierto que Hamlet fue un melancólico príncipe danés?" y respondo que no, esa persona tiene razones para creer que nunca ha leído Hamlet. Nuestra intuición sobre el valor semántico de oraciones como (1) o (2), se basa, justamente, en desafíos de ese estilo.

\section{UNA SOLUCIÓN MEdiAnte REFERENCIA DIRECTA}

En general, la propuesta de Russell es que los nombres propios, en tanto DD, poseen un contenido semántico: la descripción que va asociada. El problema señalado por Kripke es que, si mantenemos la creencia de que los nombres poseen un contenido semántico, entonces nos encontraremos con un problema. Que Aristóteles haya sido un filósofo griego es un hecho sometido a la contingencia. Podemos concebir una situación contrafáctica donde Aristóteles no haya sido filósofo. Podemos descubrir empíricamente que nada de lo que sabíamos de Aristóteles era cierto ¿Seguiremos hablando de Aristóteles si renunciamos a todas las descripciones asociadas?, ¿qué criterio usamos para seguir hablando de cierto particular dada ciertas circunstancias contrafácticas que nos alejan de toda descripción que alguna vez asociamos a él? ${ }^{18}$

Un ejercicio similar ya se puede encontrar en Nombres Propios y Descripciones, de Searle ${ }^{19}$, que concluye asumiendo que alguna de las descripciones asociadas debe ser verdadera para seguir hablando de la misma entidad. Sin embargo, Kripke irá un paso más allá y señalará que, a pesar de renunciar a todas las descripciones que asociamos normalmente a un nombre, seguiremos hablando del mismo individuo, debido a que los nombres no poseen en realidad ningún tipo de contenido semántico. A diferencia de lo que creía Russell, la función de los nombres propios es referir de manera directa y no a través de descripciones. Podemos someter a una persona cualquiera al siguiente test: si tomamos a Aristóteles y le quitamos todas las des-

18 KRIPKE, Saul. El nombrar y la necesidad. Traducción de Margarita Valdés. UNAM, 2005, pp 60-64.

19 SEARLE, J. Nombres Propios y Descripciones. En Valdés Villanueva (comp), La Busqueda del Significado. Tecnos, 2005, pp 105-114. 
cripciones que asociamos a esa persona, usted ¿lo sigue señalando a él -a la misma persona- como el referente del nombre?

Cuando decimos "es posible que Aristóteles no haya sido filósofo, sino un balsero veneciano del siglo XVIII" seguimos hablando de Aristóteles, pero sin ninguna de las características contingentes que le atribuimos. Es a Aristóteles a quien transportamos a dicho mundo posible, no a otro objeto parecido a él. Kripke concluirá que los nombres son designadores rigidos ${ }^{20}$, ya que en todo mundo posible que se pueda concebir, seguiremos hablando del mismo individuo. Si renunciáramos a esto, estaríamos desechando todo nuestra "mochila" conceptual; en toda situación contrafáctica -o mundo posible-nuestros conceptos deben seguir siendo los mismo para que podamos entender dicha situación. Después de todo, es con nuestro lenguaje y conceptos con los que estipulamos toda situación contrafáctica.

Si bien, lo planteado por Kripke coloca en aprietos la propuesta de Russell sobre los nombres propios, no nos da, a primera vista, ninguna solución respecto a EF. Sin embargo, en Nombrar y la Necesidad, nos da una pista acerca de la ontología que está teniendo en vista. Cuando tenemos enunciados como (2) y (5), podemos llegar a creer que tanto Hamlet, como los unicornios, pudieran existir en algún mundo posible, o sostener que son entidades posibles: podría ser el caso que un grupo de arqueólogos descubriera lo que parece ser un fósil de unicornio o que un grupo de historiadores descubriera que Hamlet realmente fue un príncipe danés

\begin{abstract}
Ahora bien, no hay ninguna especie real de unicornios y, con respecto a las varias especies hipotéticas diferentes con distintas estructuras internas (algunas de reptil, algunas demamífero, algunas de anfibio), las cuales tendrían las apariencias externas que, según se postula, convienen a los unicornios en el mito del unicornio, uno no puede decir cuál de entre esas especies míticas distintas habría sido la especie de los unicornios. Si suponemos, como yo lo hago, que los unicornios del mito constituían supuestamente una especie particular, pero que el mito no nos suministra una información suficiente sobre su estructura interna de manera que determine una única especie, entonces no hay ninguna especie real o posible de la que podamos decir que ésa hubiera sido la especie de los unicornios ${ }^{21}$
\end{abstract}

20 KRIPKE, Saul. El nombrar y la necesidad. Traducción de Margarita Valdés. UNAM, 2005, pp 51.

21 Ibidem, pp 153-154. 
El mito no nos otorga las pistas necesarias para poder señalar, en este mundo, a una especie de unicornios. No disponemos de la información necesaria para individuar a tales organismos. Aunque nos den una morfología externa de cómo eran los unicornios, seguiríamos sin poder señalarlos, pues es necesario conocer la estructura interna para poder señalar con certeza si es o no un unicornio. Dado que no tenemos un criterio de evaluación, no podemos establecer un escenario posible en el cual tales entidades existan. Puede ser el caso que nos encontremos con un personaje histórico que cumpla con la mayor parte de las descripciones asociadas a Hamlet cumpliendo los requisitos de Russell- pero no podemos estar seguros que en efecto sea a él a quien nos referimos cuando hablamos de Hamlet: puede ser, simplemente, una coincidencia que ambos compartan ciertas descripciones. Puede ser el caso, incluso, que Darwin se volviera detective, y cumpliera todos, o la mayoría, de las descripciones asociadas con Sherlock Holmes; pero este no es un caso donde Darwin es/fue Holmes, pues los nombres designan de manera rígida, y simplemente es un escenario donde Darwin fue detective, y no biólogo. Lo que sabemos, respecto a EF dentro de la propuesta de Kripke, es que no los podemos tomar como entidades de existencia posible.

\section{A. REFERENCIA DiReCTA Y EF}

Básicamente, si adoptamos una teoría de la referencia directa donde los nombres poseen la función de designar al objeto por el cual están, nos podemos preguntar ¿a qué refieren los nombres de EF? Tales nombres resultan vacíos, pues no poseen un objeto al cual hacer referencia. Si esto es así, entonces, ¿qué solución nos puede entregar la referencia directa?

Tal como sostenía en un inicio, y como remarcaba Meinong, sí hay un sentido en el cual (1) es verdadera. Si a un alumno se le realiza una pregunta de comprensión lectora sobre alguna obra de ficción, su respuesta está sujeta a evaluación, tiene condiciones de corrección; específicamente, si se le realiza una pregunta de verdadero y falso acerca de (1) Hamlet fue un melancólico príncipe danés, y este responde "falso", su evaluación será baja ${ }^{22}$. Esto rescata la intuición inicial: sí hay un sentido en el cual, sentencias con EF, sí son verdaderas. Sin embargo, tal sentido queda relegado a un ámbito particular, esto es, si es considerado dentro de la historia narrada.

22 KRIPKE, Saul. "Reference and Existence: The John Locke Lectures". Oxford University Press, 2013 , p 43. 
Aunque esto rescata nuestra intuición inicial respecto a tales enunciados, deja abierta la siguiente pregunta: ¿a qué hacemos referencia? Con Meinong sabíamos que referíamos a entidades subsistentes, pero ¿qué pasa con la solución de Kripke? ¿EF refiere realmente a algo? Este punto es clave, pues lo que me interesa en última instancia, como fue señalado en un comienzo, es dar con una teoría que sea justa con nuestras intuiciones, pero que a la vez sea ontológicamente desinflacionaria.

Entonces, ¿cómo abordar el problema de EF desde la referencia directa? ¿Cuál es la propuesta de fondo de Kripke? Cuando analizamos los trabajos de ficción -como las novelas- lo que se realiza es pretender que, lo sucedido en la historia narrada, está sucediendo de verdad. En tal contexto, cuando estamos en una historia, los nombres de los personajes se usan como si realmente estuvieran refiriendo a una persona real. Por ejemplo, cuando nos centramos en Hamlet, y estamos en el contexto de la obra, el nombre "Hamlet" refiere a una persona, a Hamlet. En ese contexto, el nombre "Hamlet", cumple la misma función que un nombre propio. Lo central es, justamente, tener presente que pretendemos tal situación: sólo cuando estamos en el contexto de la obra de ficción, pretendiendo que sucede una historia real, pretendemos que los nombres de los EF involucrados poseen la misma función que un nombre. Sería un error - quizás el que cometió Meinong y quienes adhieren a la realidad de EF-creer que, por pretender que los nombres de EF funcionan como nombres, tales nombres son no-vacíos.

A lo bosquejado arriba, es lo que Kripke llama el principio de pretensión (pretense

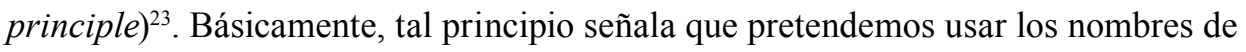
EF como si fueran no-vacíos, como si realmente refirieran a algo. Todo esto, por supuesto, en un marco y contexto determinado: la historia que está siendo narrada. Así, si usamos el nombre "Hamlet" fuera del contexto de la obra, no estamos refiriendo a nada: es un nombre vacío.

Este pretense principle nos permite mantener la parsimonia entre los nombres propios y EF, pues en ambos casos los nombres tendrían la misma función, aunque en el segundo sólo pretendamos hacer tal referencia. Algo semejante ocurre con las proposiciones involucradas: mientras (3) sería una proposición propiamente tal -en tanto posee un nombre propio con referente- (5) sería una proposición pretendida (pretended propositions) pues, como no posee las mismas circunstancias de eva-

23 KRIPKE, Saul. "Vacuous Names and Fictional Entities" Philosophical Troubles, Oxford university Press, 201, p 58. Mí énfasis 
luación, sería una instancia pretendida. De esta forma, (5) no sería una oración sin sentido, pues sabemos que proposición pretende expresar; sólo pretendemos que el nombre hace referencia a una persona, y que se dice algo acerca de esa persona ${ }^{24}$.

\section{B. Desambiguaciones}

Entonces, ¿qué pasaría con EF? La propuesta de Kripke que se ha expuesto hasta ahora, señala que pretendemos que los nombres de EF hacen referencia a algo, pero ¿a qué?

La propuesta de Kripke sostiene que EF son entidades reales, pero abstractas. Son reales en tanto la pregunta acerca de su existencia implica una pregunta acerca de nuestro mundo, concretamente, acerca de nuestras actividades. Justamente, por este último elemento, se sostiene que son abstractas: son construcciones que existen por nuestras actividades.

Si esto es así, ¿en qué sentido la propuesta dada por Kripke es distinta a la de Meinong? Como ya vimos, Meinong sostiene que EF son entidades reales subsistentes, en tanto son capaces de predicación; lo sostenido por Kripke parece, justamente, ir en una dirección similar: son entidades reales, pero abstractas, pues dependen directamente de la actividad humana, al igual que las naciones, por ejemplo. La diferencia radica, justamente, que para Kripke, tales entidades no existen, y que habría una confusión respecto a cómo entendemos la idea de EF:
When we say 'There was such a fictional character
as Hamlet', we are not referring to a ghostly person we are referring to a
fictional character, one who really does exist, because people have written works of a certain sort. As I said, fictional characters are abstract entities of a certain kind ${ }^{25}$

Al contrario de Meinong, EF no hace referencia a una entidad fantasmal, al personaje ficticio propiamente tal. Como sostenía, cuando nos centramos en la obra, los nombres propios de los EF deben ser entendidos de la misma forma que los nombres propios comunes, esto es: haciendo referencia directa a una persona. Así, lo que

24 Cfr: Ibidem, p 62

25 KRIPKE, Saul. "Vacuous Names and Fictional Entities" Philosophical Troubles, Oxford university Press, 201, pp. 64-65. Mi énfasis. 
hacemos cuando usamos sentencias como (7) Don Quijote luchó contra molinos, creyendo que eran gigantes, es referirnos a una persona, a Don Quijote; sin embargo, de manera implícita, estamos agregando un cuantificador de contexto: asumimos un contexto ficcional para hacer referencia a EF. Esto es a lo que Kripke señala como "pretender" que tales proposiciones - esto es, como el caso de (1), (5) o (7) - son proposiciones propiamente tal. Solo cuando salimos de la obra, y volvemos a sostener (7), nos damos cuenta de que Don Quijote no refiere, es un nombre vacío. Cuando sostenemos que, tales entidades son productos de nuestra actividad, estamos haciendo que estas dependan ontológicamente de nosotros.

Adicionalmente, el pretense principle señalado anteriormente, permite desmarcar la propuesta kripkeana de formas más refinadas de la propuesta de Meinong. Por ejemplo, la lectura que realiza Zalta ${ }^{26}$ sobre EF, resalta en que no poseen una existencia espacio-temporal, pero sí son objetos abstractos. Tales objetos abstractos no son de la misma índole que los señalados por Kripke, en tanto Zalta propone que deben ser entendidos como objetos genéricos, o incluso roles, siendo similares a las Formas platónicas ${ }^{27}$. Si bien hay intentos de conciliar tales teorías ${ }^{28}$, no serán tratadas acá. Sólo sostendré que, a pesar de compartir ciertos elementos en común, ambas teorías son fundamentalmente incompatibles, en tanto la propuesta de Zalta sigue sosteniendo que EF son entidades reales, independientes ontológicamente, el cual no es el caso de la ontología propuesta por Kripke, o, al menos, de lo que se puede desprender de su teoría central.

¿Qué diferencia la presente propuesta de la solución clásica de Russell? En On Denoting, Russell termina estableciendo que, enunciados como (1), son falsos, pues la Descripción Definida no es satisfecha por ninguna entidad. Llamemos tal postura anti-realista de personajes ficticios [ARPF]. Un realista de personajes ficticios [RPF] puede alegar, apelando a nuestra intuición, de que (1) es verdadera, aunque falle al referir. Si luego de leer "Don Quijote de la Mancha”, se me pregunta si Don Quijote se enfrenta con unos molinos, creyendo que eran gigantes, y yo respondo que no, la persona que me pregunta tendría razones para creer que i) no leí "Don Quijote de la Mancha”, o bien ii) lo leí, pero no presté atención, u olvidé ese pasaje en particular.

26 ZALTA, E. Abstract Objects: An Introduction to Axiomatic Metaphysics, 1983, Dordrecht: D. Reidel.

27 Cfr: Ibidem, p 41-47.

28 ZALTA, E. (2000). "The Road Between Pretense Theory and Abstract Object Theory", en Everett \& Hofweber (eds.), Empty Names, Fiction, and the Puzzles of Non-Existence, Stanford: CSLI Publications, 2000, pp. 117-147. 
Cualquiera sea la alternativa, alguien que sostiene RPF nos dirá: la creencia sobre Don Quijote está errada, la proposición es verdadera, pues es verdad que él se enfrentó con molinos creyendo que eran gigantes. Los defensores de RPF sostienen que, a partir de consideraciones semánticas, podemos llegar a conclusiones ontológicas, esto es: dado que tal proposición la podemos considerar verdadera, entonces la entidad implicada es real. Sin embargo, este desafío no implica, como sostienen los defensores de RPF, que tales entidades sean reales. En el caso de Meinong, tal y como fue expuesto, se sigue tal razonamiento: dado que podemos realizar predicaciones verdaderas, debemos concluir que la entidad en cuestión existe. Pero ese no es el caso. Lo que sí necesitamos es explicar por qué tenemos tales intuiciones sobre los personajes ficticios, a pesar de que estos no existen. Y es, justamente, en tal punto que falla $\mathrm{DD}$, pues a pesar de mantener neutralidad ontológica al respecto ${ }^{29}$, no hace justicia a una intuición base que se puede compartir con RPF: sentencias como (1) o (7) son tratadas como verdaderas, aunque no tengan referente. Esto no nos compromete, de forma alguna, con la conclusión ontológica que desean implicar RPF, pero sí demanda explicar por qué tratamos tales oraciones como verdaderas.

\section{Algunas Conclusiones}

Lo anterior es la gran virtud de la propuesta de Referencia Directa, pues, cuando sostenemos enunciados con EF como sujetos, lo hacemos dentro de un contexto determinado: la obra literaria a la cual pertenece EF. Cuando nos contextualizamos en la obra, mediante estos cuantificadores implícitos de contexto, pretendemos que los nombres de EF refieren de manera directa al objeto: "Hamlet" refiere a Hamlet. De esta forma no es problemático que, sentencias del tipo (1) Hamlet fue un melancólico príncipe danés, sean verdaderas, pues están contextualizadas en una obra determinada, y no en el mundo. En estos casos, EF actúan, nuevamente, en el sentido de pretender que, haciendo referencias a una persona, y solo una vez que nos situamos fuera de la obra, podemos sostener que no hay tal persona y que la referencia falla. De las intuiciones que deseo rescatar, el pretense principle rescata una: sentencias con personajes ficticios como sujetos, son capaces de ser verdaderas. Pero, ¿qué pasa con el aspecto ontológico? Kripke señala que EF son entidades abstractas, dependientes de nuestras actividades. Esto se puede interpretar de la siguiente manera: son entidades cuya realidad ontológica depende de nosotros, simplemente no son

29 Con esto me refiero a lo siguiente: las Descripciones Definidas de Russell no se comprometen con la existencia de la entidad, sino que se mantienen neutrales. Para ser más preciso: sólo si una entidad existe, la Descripción Definida será satisfecha, pero está no presupone la existencia de la entidad. 
independientes. Luego, podemos sostener que dependen de un contexto: cuando nos situamos dentro de una obra, podemos tratar tales entidades como si fueran entidades ontológicamente independientes, pero una vez fuera de ellas, podemos ver que tales entidades no encuentran un referente.

Semánticamente, podemos tratar tales entidades igual que los nombres propios con referencia, pero, ontológicamente -siendo este el punto que me interesa resaltar-son explicados no como entidades que existen separadas de un sujeto, sino dependientes de su actividad. Si se nos presiona y se pregunta de nuevo: ¿cómo podemos interpretar, ontológicamente, a EF? quizás podemos interpretar tales entidades como representaciones. En tal caso, cada vez que sostenemos oraciones como (1) o (7), fuera del contexto de la obra, hacemos referencia a tales representaciones, y no a una entidad capaz de existencia externa (como creía Meinong). Ahora bien, yendo un paso más allá, podríamos incluso sostener que el contenido de dichas representaciones puede ser fijado por nuestro entorno social, lo cual vendría bien con la motivación externista semántica de Kripke y sería coherente con las consecuencias que este tipo de teorías plantea respecto al contenido mental ${ }^{30}$.

Tal postura no queda exenta de problemas. El principal inconveniente es con enunciados del tipo (8) Hamlet no existe ¿Cómo tratar tal enunciado? Dado que posee como sujeto un personaje ficticio, debería ser tratado bajo el pretense principle; en ese marco, el enunciado sería falso, pues Hamlet sí existe en ese contexto, aunque sea de forma fingida. Sin embargo, sabemos que tal enunciado es verdadero: es cierto que no existe Hamlet. Frente a tal caso, ¿debemos abandonar el pretense principle? Quizás no sea necesario. Una forma de abordar enunciados como (8), sin renunciar al pretence principle, es establecer niveles de análisis del lenguaje, y aclarar lo que queremos decir con enunciados similares al anterior. Quizás lo que se quiere decir en (8) es algo como (8)* fuera del contexto de la obra, no existe Hamlet. Solo cuando analizamos (8) en un nivel distinto, y lo tomamos como (8)*, el enunciado deja de ser problemático $^{31}$. Aun así, esta no es una solución completa, y quizás no es del todo satisfactoria. El problema puede ser más extenso de lo que planteo acá.

30 Respecto al contenido mental fijado externamente, se puede remitir a BURGE, T. (1979). "Individualism and the Mental"; en Midwest Studies in Philosophy, Vol. 4, No. 1, 1979, pp 73-122, y PUTNAM, H. (1975). "The Meaning of 'Meaning", Minnesota Studies in the Philosophy of Science 7, 1975, pp 131-193.

31 Creo que el problema es mucho más extenso de lo aquí abordado, y quizás la solución no es del todo optima si se sigue presionando en esa dirección. Para mayor detalle del problema, y cómo se puede abordar desde la referencia directa, se puede remitir a KRIPKE, Saul. "Reference and Existence: The John Locke Lectures". Oxford University Press, 2013, pp 144-160. 
Por otro lado, rescatamos del desafío de RPF el reconocer que las oraciones que poseen EF como sujetos no son carentes de sentido, ni tampoco meramente falsas. Sin embargo, un defensor de RPF puede insistir, usando el mismo desafío señalado antes, y sostener que, ya que concedimos su reclamo semántico, ¿por qué no el ontológico? Aquí la solución sería otra, una menos elegante: apelar a un asunto de principios, porque aumenta, de manera innecesaria, nuestra ontología; porque tenemos otra forma de lidiar con tales entidades, la cual no implica darles un estatus ontológico independiente; porque, simplemente, no calza con un marco naturalista.

Dejando de lado las posibles complicaciones, ¿qué podemos concluir de todo esto? Primero, que no estamos en presencia de entidades fantasmales, como parecía sugerir Meinong, o cualquier otro realista de EF, pero tampoco frente a proposiciones meramente falsas como creía Russell. Estas son, justamente, las intuiciones que me interesa rescatar y compatibilizar: que sentencias con EF como sujetos sean verdaderas, pero con una ontología desinflacionaria. En ese marco, creo que la Referencia Directa es un aporte teórico fuerte para hacer justicia a nuestras intuiciones iniciales sobre EF. 


\section{Bibliografía}

Burge, Tyler. "Individualism and the Mental", en Midwest Studies in Philosophy, Vol. 4, No. 1, 1979: 73-122.

Chisholm, Roderick. (1973). "Beyond Being and Nonbeing”; en Philosophical Studies: An International Journal for Philosophy in the Analytic Tradition, Vol. 24, No. 4, 1973, pp. 245-257.

KrIPKe, Saul. El nombrar y la necesidad. Traducción de Margarita Valdés, México: UNAM, 2005.

. "Vacuous Names and Fictional Entities"; en Kripke, Saul, Philosophical Troubles, Nueva York: Oxford University Press, 2011.

. "Reference and Existence: The John Locke Lectures". Oxford University Press, 2013.

MAReK, Johann. Alexius Meinong., 2013: disponible en http://plato.stanford.edu/archives/ fall2013/entries/meinong/.

Meinong, Alexius. "The Theory of Objects". Traducción de Isaac Levi, D. B. Terrel, y Roderick Chisholm; en Realism and the Background of Phenomenology, ed. Roderick Chisholm. Ridgeview, 1981, pp. 76-117.

. "Über die Erfahrungsgrundlagen unseres Wissens" ["On the Experiential Foundations of Our Knowledge"], en Abhandlungen zur Didaktik und Philosophie der Naturwissenschaften, Band [Vol.] I, Heft [Issue] 6, Berlin: J. Springer. Reimpreso en Meinong 1968-78, Vol. V: 367-481. Epistemological treatise on empirical knowledge - on internal and external perception in contrast to a priori knowledge. Revisado por Russell, 1906.

Putnam, Hilary. (1975). “The Meaning of 'Meaning”; en Minnesota Studies in the Philosophy of Science 7 (1975): 131-193.

Russell, Bertrand. “On Denoting”; en Mind, Vol. 14, No. 56. (1905): 479-493.

SEARLe, John. "Nombres Propios y Descripciones". En Valdés Villanueva (comp), La Búsqueda del Significado, 105-114, Tecnos, 2005.

Zalta, Edward. Abstract Objects: An Introduction to Axiomatic Metaphysics, Dordrecht: D. Reidel, 1983.

. (2000). "The Road Between Pretense Theory and Abstract Object Theory", en Everett \& Hofweber (eds.), Empty Names, Fiction, and the Puzzles of Non-Existence, 117-147, CSLI Publications, 2000. 